\title{
The ALFALFA Search for (Almost) Dark Galaxies across the HI Mass Function
}

\author{
Martha P. Haynes ${ }^{1}$ \\ ${ }^{1}$ Center for Radiophysics and Space Research, Cornell University, Ithaca, NY 14853, USA \\ email: haynes@astro.cornell.edu
}

\begin{abstract}
The Arecibo Legacy Fast ALFA (ALFALFA) survey is a second generation blind extragalactic HI survey currently in progess which is exploiting Arecibo's superior sensitivity, angular resolution and digital technology to derive a census of the local HI universe over a cosmologically significant volume. As of the time of this meeting, some 4500 good quality extragalactic HI line sources have been identified in about $15 \%$ of the final survey area. ALFALFA is detecting HI masses as low as $10^{6} M_{\odot}$ and as large as $10^{10.8} M_{\odot}$ with positional accuracies typically better than $20^{\prime \prime}$, allowing immediate identification of the most probable optical counterparts. Only $3 \%$ of all extragalactic HI sources and less than $1 \%$ of detections with $M_{H I}>10^{9.5} M_{\odot}$ cannot be identified with a stellar component. Because ALFALFA is far from complete, the discussion here focuses on limitations of past surveys that ALFALFA will overcome because of its greater volume, sensitivity and reduced susceptibility to source confusion and on a sampling of illustrative preliminary results. First ALFALFA results already suggest, in agreement with previous studies, that there does not appear to be a cosmologically significant population of optically dark but HI rich galaxies. ALFALFA promises a wealthy dataset for the exploration of many issues in near-field cosmology and galaxy evolution studies, setting the stage for their extension to higher redshifts in the future with the Square Kilometer Array (SKA).
\end{abstract}

Keywords. galaxies: distances and redshifts, galaxies: dwarf, galaxies: evolution, galaxies: formation, galaxies: mass function, galaxies: spiral, (cosmology:) cosmological parameters, cosmology: observations, (cosmology:) large-scale structure of universe, radio lines: galaxies

\section{Introduction}

As described in the papers by Giovanelli, Martin and others in these proceedings, the Arecibo Legacy Fast ALFA (ALFALFA) survey is exploiting the superior sensitivity, angular resolution, spectrometer and signal processing associated with the Arecibo L-band Feed Array (ALFA) on the Arecibo 305 m antenna to conduct a "second generation" wide area blind HI survey. Following on the success of the HI Parkes All-Sky Survey (HIPASS: Zwaan et al. 2004; Meyer et al. 2004), ALFALFA offers substantial gains with respect to HIPASS (as it should!), most notably in its increased depth and angular resolution. With a median redshift of only $\sim 2800 \mathrm{~km} \mathrm{~s}^{-1}$, HIPASS did not sample adequate extragalactic volume to yield a cosmologically "fair sample" of the local universe, and the large beamsize $\left(15^{\prime}\right)$ of the Parkes telescope made identification of optical counterparts often uncertain without followup HI synthesis observations. In fact, Oosterloo et al. (2007) suggest that confusion within the large Parkes beam creates significant uncertainty in the estimate of statistical properties based on HIPASS. With 20 times smaller beam area and eight times the sensitivity, the centroiding accuracy of ALFALFA is on average $24^{\prime \prime}\left(20^{\prime \prime}\right.$ median) for all sources with signal-to-noise ratio $>6.5$, and its median redshift is nearly three times greater (Giovanelli et al. 2007). Thus, ALFALFA will produce a deeper and richer census of the local HI universe, probing a cosmologically significant volume and, in most instances, providing immediate identification of the corresponding 
optical counterpart to each HI source. Because ALFALFA is in progress, its results are not fully available. However, the currently available ALFALFA catalog contains $\sim 4500$ good quality extragalactic HI detections so that suggestive preliminary results are beginning to emerge. In the context of the present discussion of "Dark Galaxies and Lost Baryons", the most interesting objects will be ones without a clearly identified stellar component. Of the current sample, only $3 \%$ cannot be identified with a distinct optical counterpart and virtually all of the objects with $M_{H I}>10^{9.5} M_{\odot}$ can be associated with a luminous galaxy in standard imaging datasets such as SDSS or 2MASS. The majority of low mass HI detections without discrete stellar counterparts can be explained as debris remnants of a past interaction involving a nearby optically-seen galaxy (Kent et al. 2007; Haynes, Giovanelli \& Kent 2007). In this paper, I summarize the potential for ALFALFA to deliver cosmologically significant results and present some illustrative findings which the full survey promises to address in more quantitative detail.

\section{Determinations of the HI Mass Function}

One of the principal discrepancies between cold dark matter (CDM) theory and current observations revolves around the difference between the expected and observed numbers of low mass dark matter halos (Kauffmann, White \& Guiderdoni 1993; Klypin et al. 1999), sometimes referred to as the "missing satellite problem". The logarithmic slope of the faint end of the mass function predicted by CDM simulations is close to the Press-Schechter (Press \& Schechter 1974) value of $\alpha=-1.8$. Because the mass function itself is difficult to determine directly, observational efforts have mainly focused on estimates of the faint end of the optical luminosity function and its HI counterpart, the HI mass function (HIMF). By determining both, limits can be set on the number of low mass halos containing measurable stellar or gaseous components. The shape of the low mass end of the HIMF and its corollary, the cosmological mass density of HI, are important parameters in the modeling of the formation and evolution of galaxies.

The first determinations of the HIMF (e.g., Zwaan et al. 1997) used the venerable $\Sigma\left(1 / V_{\max }\right)$ method (Schmidt 1964). Application of that method assumes that the HI sources are homogeneously distributed within the sample volume, an assumption that is obviously incorrect. An alternative method for deriving luminosity and mass functions, the two-dimensional stepwise maximum likelihood (2DSWML) method proposed by Loveday (2000) has been employed by Zwaan et al. (2003) and Springob et al. (2005a). The 2DSWML method also has drawbacks in that it is not automatically normalized and assumes that the shape of the HIMF is universal. In practice, both methods are obviously limited by statistics; they can also be severely affected by systematic uncertainties, such as errors in the estimate of distances, especially nearby (Masters et al. 2004). Adopting a modified $\Sigma\left(1 / V_{\max }\right)$ approach, Rosenberg \& Schneider (2002) and Springob et al. (2005a) accounted in different ways for the effects of large scale structure by assigning an effective weight for each galaxy to account for density inhomogeneities. As the latter authors point out, a survey which does not sample adequate volume may produce unreliable results because the weighting scheme must account not only for variations of the space density of galaxies with distance but also for variations in the fraction of the surveyed volume that is occupied by regions of a particular density.

While blind HI surveys avoid issues associated with selection bias favoring high optical surface brightness targets, the fact that there does not appear to be a significant population of optically dark galaxies at least at the higher masses suggests that a significantly large and deep optically-selected sample could detect enough HI galaxies to uncover the true HIMF of spiral galaxies at least in the high mass regime. Springob et al. (2005a) 


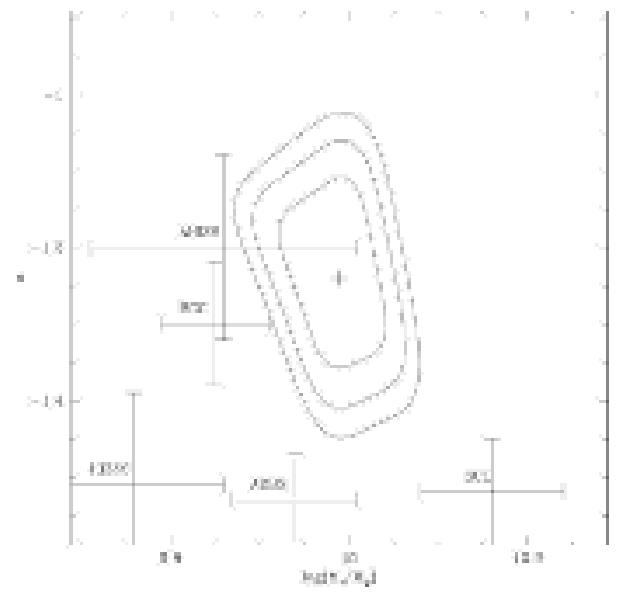

Figure 1. $\chi^{2}$ contours of the Schechter fit parameters $M_{*}$ and $\alpha$ to the HIMF for the Springob et al. (2005a) optically-selected sample. Values with error bars indicate fits derived from the HI blind surveys: HIZSS (Henning et al. 2000), BGC (Zwaan et al. 2003), AHISS (Zwaan et al. 1997), ADBS (Rosenberg \& Schneider 2002) and SCC (Kilborn 2000). See Springob et al. (2005a) for further details.

have derived the HIMF and examined its dependence on morphological type and local environment for a catalog of $\sim 8800$ galaxies detected in HI through optically-targeted observations (Springob et al. 2005b). Because of the size and depth of their sample, Springob et al. were able to identify a strictly diameter- and flux-limited subset, still with $\sim 2800$ objects in it. They used both a modified $\Sigma\left(1 / V_{\max }\right)$ method, adopting a weighting scheme based on the PSCz density field (Branchini et al. 1999), and the 2DSWML method, achieving results that agree within the derived uncertainties.

To date, determinations of the HIMF by both blind and targeted HI surveys have produced similar but not entirely reconcilable results. Figure 1 shows a comparison of the derived best-fit Schechter function characteristic HI mass $M_{*}$ and low mass end slope $\alpha$ for a selection of recent determinations of the HI mass function. The contours outline the 1-, 2- and 3- $\sigma$ fits to the Springob et al. (2005a) HIMF. The agreement between the parameter fits derived from that optically selected sample and those derived from the HI blind surveys is no worse than the internal agreement among the latter. Evident also in early results derived from limited optical redshift surveys of the local universe, discrepancies among the HIMFs derived from current HI surveys are clear symptoms of the lack of a fair sample. While optically selected samples may exclude gas rich, low optical luminosity and low surface brightness galaxies, the HI blind surveys preceding ALFALFA have sampled a much shallower volume than typical optical surveys. The sampling of insufficient depth raises the potential for systematic errors in the HI mass estimates due to distance uncertainties and in the selection of a population of galaxies that is not representative of the global population detected at larger distances. For example, Masters et al. (2004) have shown that the slope of the HIPASS HIMF is actually steeper than reported by Zwaan et al. (2003) when the effects of peculiar velocities in the surveyed volume are properly taken into account. Furthermore, the lack of sufficient sampling of galaxies with very high $\mathrm{HI}$ masses, $M_{H I}>10^{10} M_{\odot}$, reduces the predictive power of past surveys to expectations for future surveys of $\mathrm{HI}$ emission from galaxies at moderate to high redshift, as discussed in Section 5. 

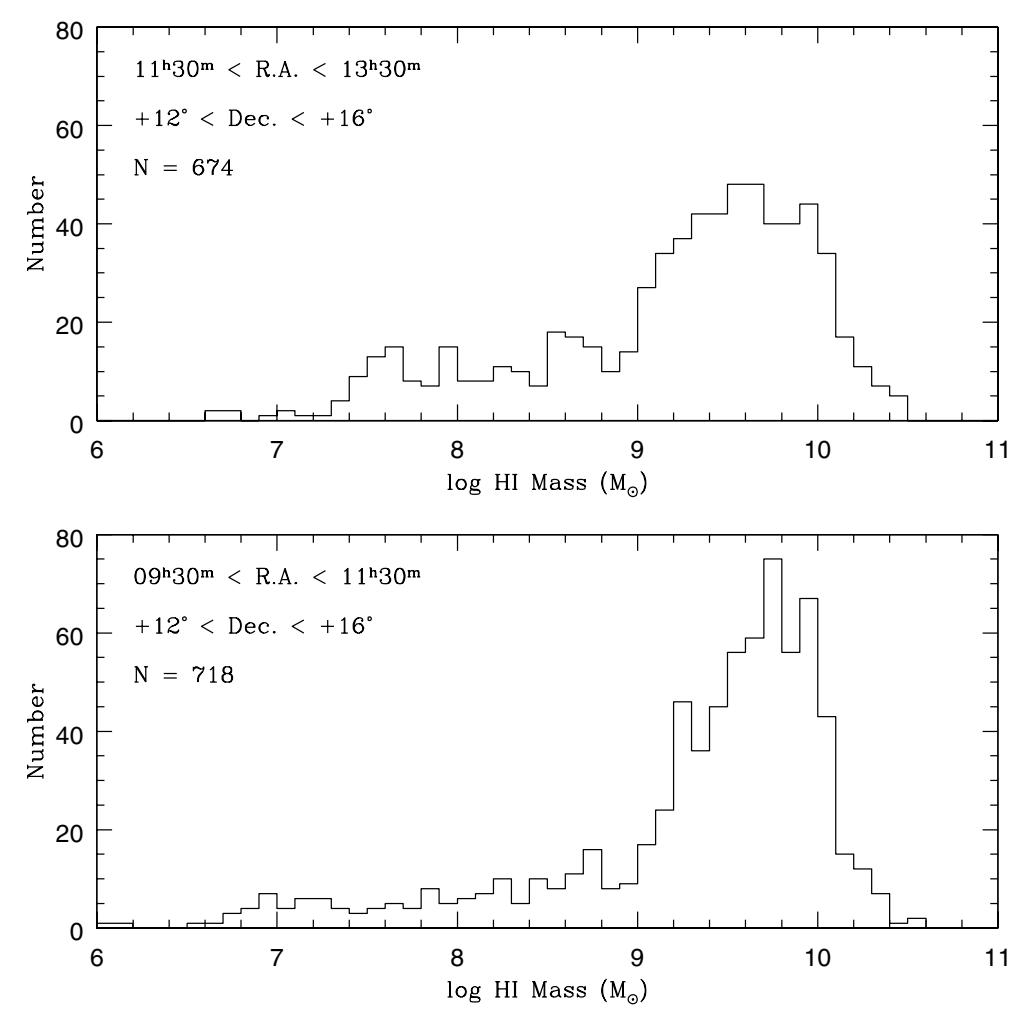

Figure 2. Left: Upper: Histogram of the HI masses for ALFALFA sources in the region $11^{h} 30^{m}<$ R.A. $<13^{h} 30^{m},+12^{\circ}<$ Dec. $<+16^{\circ}$ including the Virgo cluster region. All objects with $\mathrm{c} z<3000 \mathrm{~km} \mathrm{~s}^{-1}$ and found within $5^{\circ}$ of M87 are placed at the Virgo distance of $16.7 \mathrm{Mpc}$.. Lower: Similar histogram over the same declination band but in the range $09^{h} 30^{m}<$ R.A. $<11^{h} 30^{m}$.

\section{The Low Mass End of the HIMF}

Previous determinations of the HIMF below $10^{8} M_{\odot}$ (Zwaan et al. 1997; Rosenberg \& Schneider 2002; Zwaan et al. 2003) suffer severely from small number statistics and from the systematics associated with distance uncertainties and large scale inhomogeneities. As discussed in Section 4 of Giovanelli et al. (2005a), the most advantageous approach to increasing the number of detections of a given HI mass (once such a mass is detectable at an astrophysically interesting distance) in a survey of fixed observing time is to increase the solid angle of the survey, not its depth. Hence the Arecibo Galaxy Environments Survey (AGES) discussed by Minchin in this volume, will have a median redshift 1.5 times that of ALFALFA and will explore richly selected regions of nearby clusters and groups, but AGES will detect far fewer low mass objects than ALFALFA will because it covers only $1 / 35$ th as much solid angle.

ALFALFA is specifically designed to survey enough solid angle to detect several hundred objects with $M_{H I}<10^{7.5}$ and thus provide a robust determination of the low HI mass slope of the HIMF. Already, ALFALFA has detected more galaxies with masses less than $M_{H I}<10^{7.5} M_{\odot}$ than included in all of the previous HI blind surveys combined.

A major issue confronting the determination of the low mass end of the HIMF arises from the errors inherent in distances derived from redshifts alone, even when sophisticated flow models based on redshift-independent distances are used (Masters et al. 2004). Using the current ALFALFA dataset, Figure 2 illustrates the resultant problem. The upper 


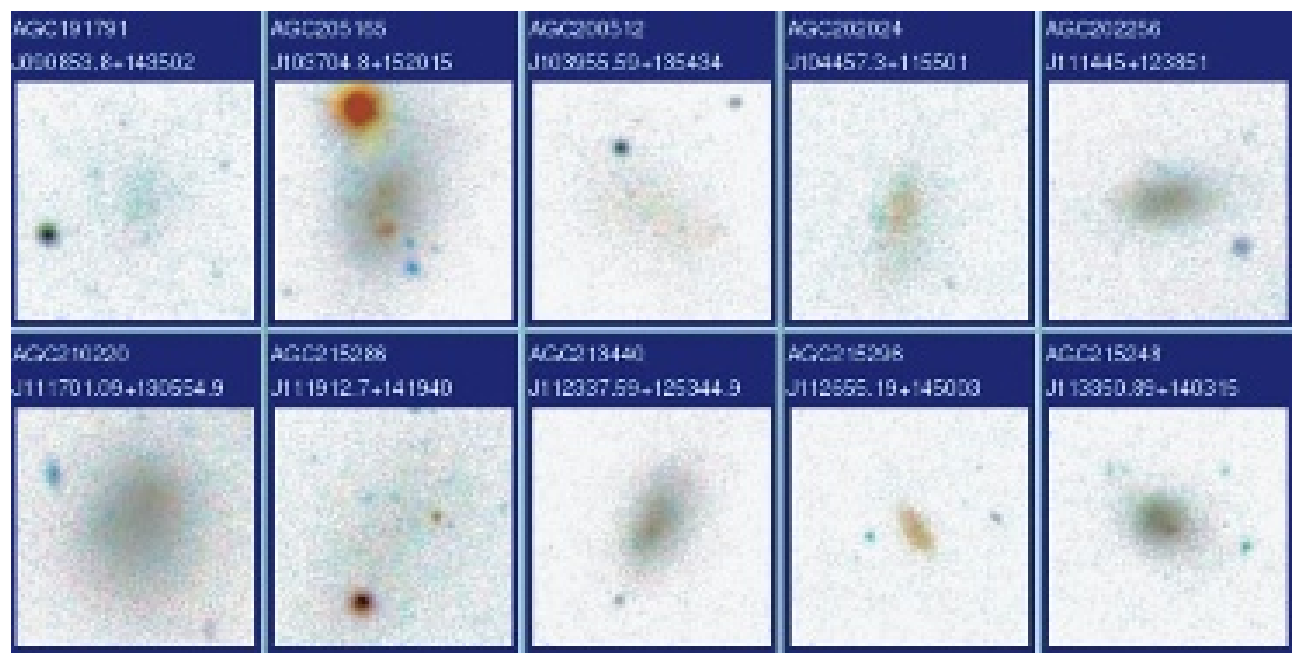

Figure 3. SDSS images of the optical counterparts of representative lowest HI mass $\left(M_{H I}<10^{7.2} M_{\odot}\right)$ ALFALFA detections. Each image is $50^{\prime \prime}$ square and uses standard SDSS Sky Server scaling.

panel shows the histogram of HI masses contained in a $120 \mathrm{deg}^{2}$ region from $11^{h} 30^{m}<$ R.A. $<13^{h} 30^{m},+12^{\circ}<$ Dec. $<+16^{\circ}$. There is no restriction placed on redshift, but galaxies found within $5^{\circ}$ of M87 and with $\mathrm{c} z<3000 \mathrm{~km} \mathrm{~s}^{-1}$ are assumed to be members of the Virgo cluster and are assigned distances of $16.7 \mathrm{Mpc}$. Elsewhere, a flow model derived from a combination of primary and secondary distances is used (Masters 2005). Very few galaxies are detected with $M_{H I}<10^{7.5} M_{\odot}$. For comparison, the lower panel shows a similar histogram for a contiguous equal area extending from $09^{h} 30^{m}<$ R.A. $<$ $11^{h} 30^{m}$ at the same declinations. The difference in the histograms is striking. At higher masses, the differences can be ascribed to the known HI deficiency of galaxies in Virgo and cosmic variance in the large scale structure sampled by the two regions. However, it is likely that the apparent deficit of galaxies at lowest HI mass and the bulge at $M_{H I} \sim 10^{7.7} M_{\odot}$ seen in the upper panel can be explained in part by the incorrect assignment of distances to some of the galaxies in the vicinity of Virgo. If some of them actually lie in the foreground, then their masses are overestimated; use of the correct, nearer distances would shift some of the objects in the peak to fill in those "missing" at lower masses. Future work to obtain more precise distance estimates for these objects is clearly in order both to explore physical mechanisms which may alter the HIMF locally and to understand better the large scale structure in and around the Virgo cluster.

\subsection{The Low Mass Galaxies as (Almost) Dark Galaxies}

In addition to providing a robust determination of the faint end slope of the HIMF, ALFALFA promises to identify a sample of nearby, low mass dwarf galaxies which can be identified from more massive but gas-poor systems because of their small HI line widths. Figure 3 shows the SDSS images of a representative set of the lowest HI mass systems, with $M_{H I}<10^{7.2} M_{\odot}$. All of them are nearby, optically faint and of low surface brightness. They exhibit a range of morphological clumpiness and evidence of massive star formation.

For her Ph.D. thesis, Amélie Saintonge (2007a,b) has undertaken a first study of some of the lowest HI mass galaxies detected early in the ALFALFA survey. Various members of the ALFALFA consortium are pursuing multiwavelength observations including 

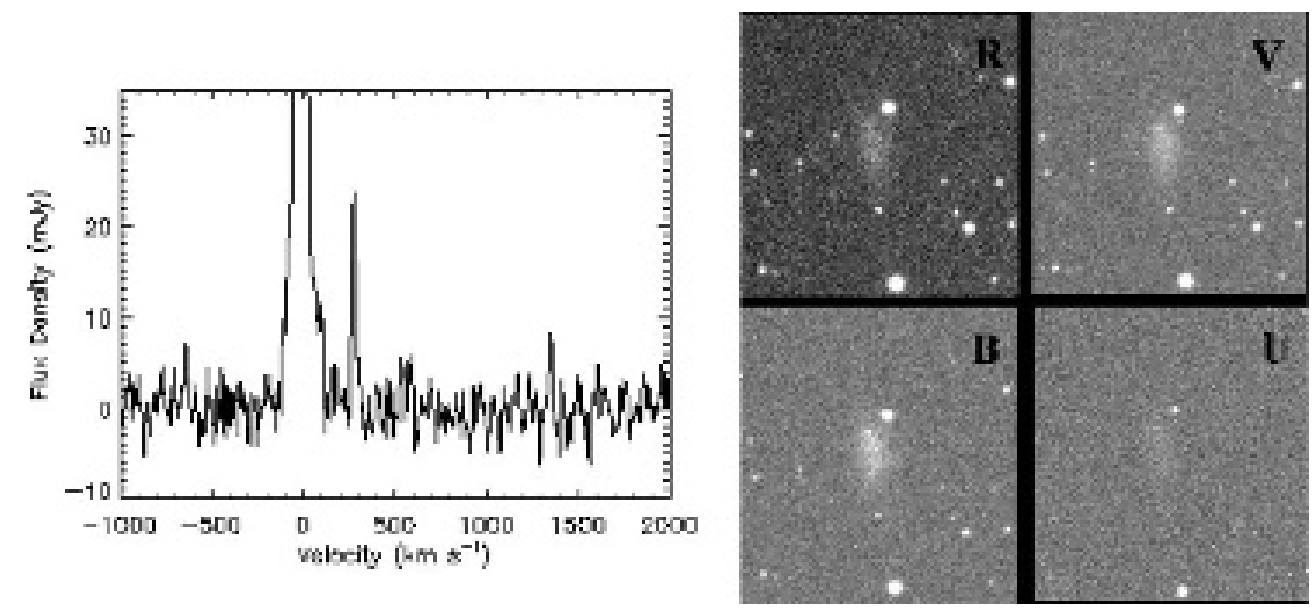

Figure 4. Left: ALFALFA spectrum of AGC 112521 (HI0141+27). Right: Broad-band images (courtesy of L. van Zee) using the WIYN 0.9 m telescope of the optical counterpart, a newly-discovered low surface brightness dwarf member of the NGC 672 group.

broad-band and $\mathrm{H} \alpha$ imaging, HI synthesis mapping and UV imaging with the GALEX satellite. Of a first sample targeted for follow-up $\mathrm{H} \alpha$ imaging, 15\% do not show the presence of HII regions. While this work is still on-going, we present an illustrative example of the kind of newly catalogued system that ALFALFA finds. Figure 4 shows the ALFALFA HI spectrum of a new dwarf member of the NGC 672 group dubbed AGC 112521. This object was first detected in our ALFA-commissioning precursor observations (Giovanelli et al. 2005b) and recovered in the early ALFALFA survey observations. As evident in Figure 4, the HI line emission from the galaxy is clearly detected at $\mathrm{c} z=+274 \mathrm{~km} \mathrm{~s}^{-1}$, a narrow line full width at $50 \%$ of the peak emission of $26 \mathrm{~km} \mathrm{~s}^{-1}$ and a total $\mathrm{HI}$ line flux density of $0.68 \mathrm{Jy}^{\mathrm{km} \mathrm{s}}{ }^{-1}$ (Saintonge et al. 2007). Assuming its membership in the NGC 672 group at a distance of $7.2 \mathrm{Mpc}$ (Sohn \& Davidge 1996; Karachentsev et al. 2004), the HI mass is $7.9 \times 10^{6} M_{\odot}$. Its blue luminosity $\mathrm{L}_{B}$ derived from newly acquired images is similarly low $\mathrm{L}_{B}=3.6 \times 10^{6} L_{\odot}$, so that $M_{H I} / \mathrm{L}_{B}$ is 2.18 . Adopting standard relations to convert multiband magnitudes into stellar mass, we find that nearly half of the baryonic matter is still in the form of gas.

With the promise of a final catalog of thousands of galaxies in the mass range $10^{6}<$ $M_{H I}<10^{8} M_{\odot}$, the study of these gas rich systems is a prime science driver of ALFALFA. Future ALFALFA studies will explore not only the low mass slope of the HI mass function and its possible dependence on environment, but also the distribution, morphology, star formation rate and chemical enrichment history of these low mass gas-rich dwarfs.

\section{The Environment and Clustering of Gas-Rich Galaxies}

In addition to the missing satellite problem, the behavior of the HIMF with varying galaxy environment has a direct bearing on the use of the HI line for cosmological purposes and on fundamental issues of galaxy formation and evolution. Early studies of the possible environmental dependence of the HIMF were limited to comparisons of the HIMF derived for galaxies in the Virgo cluster with that derived for galaxies in the field (Hoffman et al. 1992; Briggs \& Rao 1993; Rosenberg \& Schneider 2002; Davies et al. 2004; Gavazzi et al. 2004). While all of these studies suffer to varying degrees from poor statistics and incompleteness, their results marginally suggest that the HIMF in 
Virgo is missing the low HI mass dwarfs found in the field or is at least flatter at the faint end than the field HIMF. Exploiting the large and deep optically-selected sample of Springob et al. (2005b), Springob et al. (2005a), divided the sampled volume into density regimes based on the PSCz (Branchini et al. 1999) density field and compared the derived HIMFs in different regimes. They concluded that the low mass slope $\alpha$ is shallower and $M_{*}$ lower in high density environments, in qualitative agreement with the suggestions of the earlier studies based on Virgo alone. Zwaan et al. (2005) also looked at the environmental dependence of the HIMF and came to the opposite conclusion: that the HIMF is steeper in high density regions. However, those authors use a local density estimator that is purely HIPASS-based, that is, reflective of the density field of the survey itself, with no corrections for peculiar velocities or bias. Other recent work, based on the Canes Venatici region, confirms the earlier findings that the low mass slope is shallow in that region (Kovač, Oosterloo \& van der Hulst 2005). A much larger sample with many more galaxies over a larger volume is required in order to allow an investigation of whether the HIMF shape is dependent on morphological type and environment separately, as has been done by Croton et al. (2005) for the 2dF Survey optical luminosity function. Clearly ALFALFA will contribute significantly to a firmer understanding of the interplay between environment and the HIMF.

Although they acknowledge the volume and resolution limitations of the HIPASS catalog, recent papers have explored the behavior of the galaxy distribution it traces by attempting to estimate its two-point correlation function $\xi(r)$. Meyer et al. (2007) conclude that gas-rich galaxies are among the most weakly clustered galaxies known and suggest that the clustering scale length $r_{o}$ depends strongly on rotational velocity, and thus, by implication, on the halo mass. In contrast, Basilakos et al. (2007) argue that massive HIPASS galaxies show the same clustering properties as optically-selected ones, but that the low mass systems, $M_{H I}<10^{9} M_{\odot}$, show a nearly uniform distribution. Both of these studies are fraught with potential systematics because of the shallow depth of HIPASS, the potential impact of source confusion and the statistical limitations when its catalog is divided into subsets. As the follow-on to HIPASS, ALFALFA is specifically designed to overcome many of the limitations of the earlier survey, thereby allowing a robust determination of the HI-HI and HI-optical galaxy correlation functions and a quantitative study of the biasing of the HI population relative to optical or IR-selected samples and to the underlying density field.

\subsection{The Void Problem}

As pointed out by Peebles (2001), numerical simulations based on CDM predict that voids should contain large numbers of dwarf galaxies. Peebles suggests that the failure to identify such a void population raises one of the principal challenges to CDM models. For example, the voids in the simulations of Gottlöber et al. (2003) are criss-crossed by dark matter filaments, within which lie large numbers of very low amplitude inhomogeneities. In fact, those authors predict that a void with a diameter of $20 h^{-1} \mathrm{Mpc}$ should contain 1000 dark matter halos with masses of $10^{9} M_{\odot}$ and as many as 50 with masses ten times greater than that. Photoionization and baryonic blowout may suppress star formation or perhaps the retention of any baryons within the low mass halos, but it is not at all clear that these processes are sufficient to explain the absence of galaxies in voids (Hoeft et al. 2006).

Limits on the abundance and properties of void galaxies have been placed by previous optical and radio studies. Hoyle et al. (2005) have used the SDSS dataset to show that the luminosity function of void galaxies has a fainter break luminosity $\mathrm{L}^{*}$ but a similar faint end slope to the overall SDSS luminosity function. In addition, they have shown that 
void galaxies are typically blue, disk-like and have high $\mathrm{H} \alpha$ equivalent widths, making them excellent targets for HI emission line surveys.

Previous surveys for HI in voids have exploited the VLA to conduct blind HI surveys of the Pisces-Supercluster and its foreground void (Weinberg et al. 1991; Szomoru et al. 1994) and the Bootes void (Szomoru et al. 1993; 1996). Szomoru et al. (1993) did find an isolated galaxy in Bootes, but its mass of $5 \times 10^{9} M_{\odot}$ and blue luminosity $\mathrm{L}_{B}$ of -18.6 exclude it as a true dwarf. In fact, those VLA surveys sampled a relatively small volume and were hampered in the detection of low mass objects by poor spectral resolution (42 $\mathrm{km} \mathrm{s}^{-1}$ ). More recently, Pustilnik et al. (2002) have explored the HI content of a sample of blue compact galaxies known to be located in voids and find them to be "darker", as measured by their HI mass to blue luminosity ratios $M_{H I} / L_{B}$ than their counterparts in higher density regions.

Saintonge et al. (2007) initiated a first, but limited, ALFALFA analysis of galaxies in a portion of the nearby void in front of the Pisces-Perseus supercluster at $c z \sim 2000$ $\mathrm{km} \mathrm{s}^{-1}$. They detected no galaxies in a large volume of $460 \mathrm{Mpc}^{3}$, whereas a scaling of the predictions of Gottlöber et al. (2003) under the assumption that the dark-to-HI mass ratio is 10:1 predicts that ALFALFA would have detected $38 \mathrm{HI}$ sources. This very preliminary result for a single void in only $2 \%$ of the ALFALFA survey suggests that the discrepancy between the predicted and observed abundance of dwarf galaxies in voids cannot be reconciled by a population of gas rich dwarfs. More credible results will be available when the full ALFALFA survey is completed.

\section{The High Mass End: Prelude to the SKA}

One of the prime science drivers of the Square Kilometer Array (SKA) is the undertaking of a billion galaxy redshift survey in the HI line over the redshift range $0<z<2.5$ to explore the evolution of the gas content of galaxies and constrain the dark energy equation of state through the measurement of baryon acoustic oscillations (Abdalla \& Rawlings 2004). Even allowing for the likely increase in the gas content with $z$, only the most massive HI galaxies will be detected in emission at moderate redshift. And, as in the case of the low mass end of the HIMF, previous surveys have been too shallow to detect the most massive HI galaxies. ALFALFA has already detected more than twice as many massive galaxies with $M_{H I}>10^{10.4} M_{\odot}$ than all the previous HI blind surveys combined. Figure 5 shows SDSS images of the optical counterparts of a representative set of these massive ALFALFA detections. They exhibit a range of morphologies, colors and nuclear concentration but all appear to be luminous disk systems. Many of these have stellar masses in the range corresponding to the "transition mass" ( $M_{\text {stars }} \sim 3 \times 10^{10}$ $\left.M_{\odot}\right)$ above which galaxies show a marked decrease in their present to past-averaged star formation rates (Kauffmann et al. 2003).

ALFALFA will contribute its high mass detections to the GALEX-Arecibo-SDSS Survey (GASS; P.I.: D. Schiminovich) which aims to obtain measures of the HI content at a fixed gas mass fraction (1.5\%) of the stellar mass in a sample of 1000 galaxies chosen by optical-UV criteria to have stellar masses $>10^{10} M_{\odot}$. The combination of multiwavelength data will provide new understanding of the physical processes that regulate gas accretion and its conversion into stars in massive systems. HI can currently be detected in emission from normal galaxies out to a redshift approaching $z \sim 0.3$. The highest redshift detection of a single object to date, reported by Catinella in this volume, is $z \sim 0.28$; such observations require several hours of telescope time with Arecibo today. While ALFALFA+GASS will characterize the properties of galaxies at $z \sim 0$, ambitious future studies aimed at characterizing the evolution of galaxies over the last 4 Gyr should 


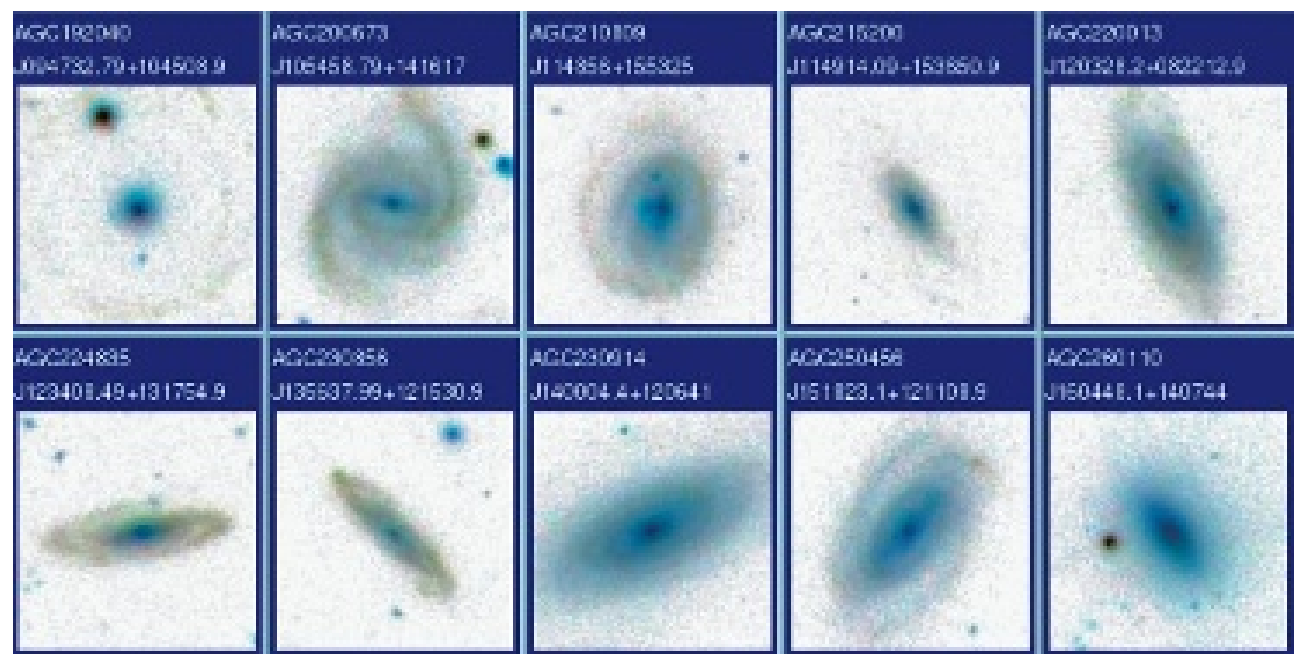

Figure 5. SDSS images of the optical counterparts of representative highest HI mass $\left(M_{H I}>10^{10.4} M_{\odot}\right)$ ALFALFA detections. Each image is $50^{\prime \prime}$ square and uses standard SDSS Sky Server scaling. Massive HI galaxies such as these will be detected in large numbers by planned future SKA surveys.

be possible in the next few years with Arecibo as well as the SKA precursor instruments under construction today.

\section{Conclusions}

ALFALFA is an ongoing survey with a detection catalog available to us at the time of this conference amounting to only about $15 \%$ of the final survey. Given its state, the full impact of ALFALFA is only beginning to become evident, but the survey promises now to yield > 25000 extragalactic HI detections when it is complete. It should yield robust measures of the HIMF, the HI-HI and HI-optical correlation functions and their bias parameters at $z=0$, thereby laying a firm footing for future studies of their evolution over cosmic time. ALFALFA is an open consortium and interested parties are invited to follow the survey's progress via the ALFALFA website http://egg.astro.cornell.edu/alfalfa.

\section{Acknowledgements}

This work has been supported by NSF grants AST-0307661, AST-0435697 and AST0607007 and by the Brinson Foundation. The Arecibo Observatory is part of the National Astronomy and Ionosphere Center which is operated by Cornell University under a cooperative agreement with the National Science Foundation. Funding for the SDSS and SDSS-II has been provided by the Alfred P. Sloan Foundation, the Participating Institutions, the National Science Foundation, the U.S. Department of Energy, the National Aeronautics and Space Administration, the Japanese Monbukagakusho, the Max Planck Society, and the Higher Education Funding Council for England. The SDSS Web Site is http://www.sdss.org/.

\section{References}

Abdalla, F. B. \& Rawlings, S. 2005, MNRAS 360, 27

Basilakos, S., Plionis, M., Kovač, K. \& Voglis, N. 2007, MNRAS 378, 301

Branchini, E. et al. 1999, MNRAS 308, 1 
Briggs, F. H. \& Rao, S. 1993, ApJ 417, 494

Croton, D. J. et al. 2005, MNRAS 356, 1155

Davies, J. I. et al. 2004, MNRAS 349, 922

Gavazzi, G., Boselli, A., van Driel, W. \& O'Neil, K. 2004, A\&A 429, 439

Giovanelli, R. et al. 2005a, AJ 130, 2598

Giovanelli, R. et al. 2005b, AJ 130, 2613

Giovanelli, R. et al.. 2007, $A J$ 133, 2569

Gottlöber, S., Łokas, E. L., Klypin, A. \& Hoffman, Y. 2003, MNRAS 344, 715

Haynes, M. P., Giovanelli, R., \& Kent, B. R. 2007, ApJL 665, L19

Henning, P. A. et al. 2000, AJ 119, 2686

Hoeft, M., Yepes, G., Gottlöber, S. \& Springel, V. 2006, MNRAS 371, 401

Hoffman, G. L., Salpeter, E. E., Lamphier, C. \& Roos, T. 1992, ApJL 388, L5

Hoyle, F., Rojas, R. R., Vogeley, M. S. \& Brinkmann, J. 2005, ApJ 620, 618

Karachentsev, I. D., Karachentseva, V. E., Huchtmeier, W. K. \& Makarov, D.I. 2004, AJ 127, 2031

Kauffmann, G., White, S. D. M. \& Guiderdoni, B. 1993, MNRAS 264, 201

Kauffmann, G. et al. 2003, MNRAS 346, 1055

Kent, B. R. et al. 2007, ApJL 665, L15

Kilborn, V. A. 2000, Ph.D. thesis, U. Melbourne

Klypin, A., Kratsov, A. V., Valenzuela, O. \& Prada, F. 1999, ApJ 522, 82

Kovač, K., Oosterloo, T. A. \& van der Hulst, J.M. 2005, astro-ph/0508072

Loveday, J. 2000, MNRAS, 312, 557

Masters, K. L., Haynes, M. P. \& Giovanelli, R. 2004, ApJL 607, L115

Masters, K. L. 2005, Ph.D. thesis, Cornell University

Meyer, M. J. et al. 2004 MNRAS 350, 1195

Meyer, M. J. et al. 2007 ApJ 654, 702

Oosterloo et al. 2007 A\&SA 465, 787

Peebles, P. J. E. 2001, ApJ 557, 495

Press, W. \& Schechter, P. L. 1974, ApJ 187, 425

Pustilnik, S. A., Martin, J. -M., Huchtmeier, W. K., Brosch, N., Lipovetsky, V.A. \& Richter, G.M. 2002 A\& A 389, 405

Rosenberg, J. \& Schneider, S. E. 2002, ApJ 567, 247

Saintonge, A. 2007a, AJ 133, 2087

Saintonge, A. 2007b, Ph.D. thesis, Cornell University

Saintonge, A. et al. 2007, AJ submitted

Schmidt, M. 1968 ApJ 356, 1155

Sohn, Y. -J. \& Davidge, T. J. 1996, AJ 112, 2559

Springob, C. M., Haynes, M. P. \& Giovanelli, R. 2005a, ApJ 621, 215

Springob, C. M., Haynes, M. P., Giovanelli, R. \& Kent, B. R. 2005b, ApJS 160, 149

Szomoru, A., van Gorkom, J. H., Gregg, M .D. \& de Jong, R. S. 1993, AJ 105, 464

Szomoru, A. et al. 1994, AJ 108, 491

Szomoru, A., van Gorkom, J. H., Gregg, M. D. \& Strauss, M. A. 1996, AJ 111, 2150

Weinberg, D. H., Szomoru, A., Guhathakurta, P. \& van Gorkom, J. H. 1991, ApJL 372, L13

Zwaan, M., Briggs, F. H., Sprayberry, D. \& Sorar, E. 1997, ApJ 490, 173

Zwaan, M. et al. 2003, AJ 125, 2582

Zwaan, M. et al. 2004, MNRAS 350, 1210

Zwaan, M. et al. 2005, MNRAS 359, 30 